This item was submitted to Loughborough's Research Repository by the author.

Items in Figshare are protected by copyright, with all rights reserved, unless otherwise indicated.

\title{
Evaporation of sessile droplets
}

PLEASE CITE THE PUBLISHED VERSION

http://dx.doi.org/10.1016/j.cocis.2014.07.005

\section{PUBLISHER}

(c) Crown Copyright. Published by Elsevier

\section{VERSION}

AM (Accepted Manuscript)

\section{PUBLISHER STATEMENT}

This work is made available according to the conditions of the Creative Commons Attribution-NonCommercialNoDerivatives 4.0 International (CC BY-NC-ND 4.0) licence. Full details of this licence are available at: https://creativecommons.org/licenses/by-nc-nd/4.0/

\section{LICENCE}

CC BY-NC-ND 4.0

\section{REPOSITORY RECORD}

Kovalchuk, Nina, Anna Trybala, and Victor Starov. 2017. "Evaporation of Sessile Droplets". figshare. https://hdl.handle.net/2134/25771. 


\title{
Evaporation of sessile droplets
}

\author{
Kovalchuk N.M. ${ }^{a, b}$, Trybala A. ${ }^{a}$, Starov V.M. ${ }^{a *}$ \\ aDepartment of Chemical Engineering, Loughborough University, Loughborough \\ LE 11 3TU, UK \\ bInstitute of Biocolloid Chemistry, 03142 Kiev, Ukraine
}

\begin{abstract}
Recent developments in the studies of evaporation of liquid droplets placed on a solid substrate are reviewed for the droplet size typically larger than $1 \mu \mathrm{m}$, so that kinetics effects of evaporation are neglected. The attention is paid to the limits of applicability of classical diffusion model of evaporation, effect of substrate, evaporation of complex fluids and applicability for its description of the theory developed for pure liquids, and hydrothermal waves accompanying evaporation.
\end{abstract}

Keywords: kinetics of evaporation, wetting, contact angle, surfactant solution, nanosuspension, Marangoni effect.

*Corresponding author 


\section{Introduction}

Evaporation of liquid droplets is omnipresent in our everyday life and in many industrial processes. The examples are cooling and combustion, using sprays in painting, cosmetics and drug delivery etc. (see, for example, Refs. [1,2] and references therein). There is a lot of publications on the evaporation of free suspended droplets (see Refs. [2,3] for review), but the problem complicates considerably for the droplets placed on a solid substrate, because in this case the mass transfer between liquid and vapour is coupled with the heat transfer between three phases involved. Moreover, solid/liquid interaction comes into play and the wetting properties of liquid should be taken into account [1]. In the case of droplets on a solid substrate the early stage of evaporation is coupled with spreading process, whereas later evaporation can cause dewetting of solid [4*]. A very comprehensive review on evaporation of droplet of pure liquids, covering more than century of research in the field, was published very recently [5*].

The further complication appears for complex liquids, such as surfactant solutions and nano-fluids, as the interactions with constituents of those liquids becomes important [6*]. The evaporation of sessile droplets of complex liquids is accompanied by self-assembly and self-organization processes resulting in formation of homogeneous or nano- and micro- structured patterns on solid interface [7,8*]. Patterned solid substrates are widely used in industrial processes, such as printing, patterning, fabrication of MEMS (microelectromechanical systems), memory and microelectronic devices, separation of polymers, agrochemistry, and micro- and nanofluidics. Bottom-up techniques of patterning [9], based on self-assembly and self-organization processes are expected to be cheaper and less energy consuming than most of existing top-down techniques. That is why the understanding and control of evaporation of complex liquids are of great scientific and industrial importance and attract considerable scientific attention.

In this review we discuss the most recent results of study on evaporation of liquid droplets on solid substrates published mainly during the last 3 years (some important results from the previous years are included as well) with special attention paid to the limits of applicability of classical diffusion model of evaporation, evaporation of complex fluids and hydrothermal waves accompanying evaporation.

\section{Dynamic regimes and kinetics of simultaneous spreading and evaporation}

The classical model for theoretical study of evaporation of liquid droplets on solid substrate is based on the assumptions of diffusion controlled mass transfer in gas phase and constant temperature over the whole system (isothermal conditions) $\left[10^{\star \star}, 11^{\star \star}\right]$. In this case evaporation kinetics is governed by the equation: 


$$
\frac{\mathrm{d} m}{\mathrm{~d} t}=\dot{m}=-\pi \mathrm{DL} \Delta c F(\theta),
$$

where $m$ is the droplet mass, $t$ is the time, $D$ is the vapour diffusion coefficient, $\Delta c$ is the vapour concentration difference between the liquid surface (saturated vapour, $c_{s}$ ) and ambient air far from the droplet, $F(\theta)$ is the function of the contact angle $\theta$ (see $\left[4^{*}, 12^{\star *}\right]$ for details and references), $L$ is the contact line radius. It should be stressed that although the evaporation flux has maximum near the three phase contact line (in the case $\theta<90^{\circ}$ ), the proportionality of evaporation rate to the droplet perimeter, but not its surface, is merely the consequence of the diffusion controlled evaporation $\left[4^{*}, 11^{\star *}, 13^{*}\right]$.

The kinetic mechanisms can be rate controlling in the case of small droplets, smaller than $1 \mu \mathrm{m}$ [14]. However in what follows only the results for larger droplets are considered and therefore the kinetic effects are neglected.

\subsection{Violation of isothermal conditions}

The most important contribution made recently to the model Eq. (1) was introducing the thermal effects due to evaporative cooling $\left[12^{\star *}, 13,15^{\star}-20^{\star}\right]$, which slow down the rate of evaporation, i.e. consideration the fact that the surface temperature $T_{\text {surf }}$ differs from the ambient temperature $T_{\infty}$. The deviations from isothermal case $\left(T_{\text {surf }}\right.$ $\left.=T_{\infty}\right)$ is considered in $\left[12^{\star *}\right]$ in terms of introduced here dimensionless SefianeBennacer (SB) number. The results of this study are presented in Fig.1 showing in particular, that the evaporation slows down by an increase of the latent heat of evaporation and the substrate thickness as well as by a decrease of the substrate thermo conductivity. The effect of the substrate thermo conductivity has been confirmed by direct numerical simulations of droplet evaporation for both pinned and de-pinned contact line $\left[15^{\star}, 16^{* *}, 17^{\star}\right]$. The theoretical predictions are in good agreement with the experimental results presented in $\left[17^{*}, 18\right]$. It is interesting that despite of much more intensive evaporation near the three phase contact line, the temperature here is the highest one over the droplet surface [19]. The reason is that the surface at the contact line has the best conditions to get energy from the substrate. The detailed consideration performed in [20*] displayed that using the

average surface temperature $T_{\text {surf }}=\frac{1}{S} \int_{S} T_{S} d S$ ( $S$ is the surface area, $T_{S}$ is the local temperature related to each surface point) enables to bring to one universal curve the numerical data on evaporation of droplets on substrates of various thermo conductivities.

\subsection{Convection in the vapour phase}

The model represented by Eq. (1) assumes diffusion controlled vapour transfer and neglects the effect of convection in vapour phase. Good agreement of the theoretical 
predictions with experimental results displayed, for example, in [16**, 21] for water on various substrates and in [2*] for the wide range of conditions (five liquids on four coatings providing contact angles from 17 to 134 degrees), can be considered as a confirmation of the validity of this assumption. However, the deviation of the experimental results from the isothermal diffusion-controlled model starts to grow, if the substrate temperature deviates from the room temperature [23]. To be precise, for metallic substrates the diffusion model underestimates the evaporation rate. The possible reason of this deviation is the increasing importance on thermal buoyancy convection at higher temperatures. The results of numerical simulations presented in [24*] show that the diffusion model underestimates the evaporation rate by $8.5 \%$ at the substrate temperature $25{ }^{\circ} \mathrm{C}$ (equal to ambient temperature) and by $27.3 \%$ at the substrate temperature $70^{\circ} \mathrm{C}$.

Several other researches have been performed during last years to outline the range of experimental conditions wherein the problem remains diffusion controlled. Two parallel sets of experiments have been performed in the terrestrial and microgravity conditions $\left[25^{\star \star}\right]$ displaying clearly that the deviations of the experimental results from the model is due to buoyancy driven effects (Fig. 2). The difference between the experimental results and the model predictions is rather small if the substrate temperature is close to the ambient temperature, but it increases considerably by an increase of the temperature difference. According to [26] for the substrate kept at the room temperature the deviation of experimental results from the diffusion model increased with the increase of the contact line radius in the range of $1-25 \mathrm{~mm}$. The effect of buoyancy convection on the droplet evaporation was studied in [27] for hexane and 3-methylpentane at the room conditions. It was shown that the vapour distribution around the droplets of both substances deviated noticeably from the spherical profile expected by diffusion controlled mass transfer.

The effect of forced convection on the kinetics of evaporation of a methanol droplet on a Teflon substrate has been studied in [28]. As it was stressed in [28] the intensive air motion can modify not only the mass transfer on liquid/air interface, but also the local temperature distribution on the surface, what results in the rather complicated time dependence of the droplet contact angle, including a stage when the contact angle increases. In [28] this increase was related to a noticeable decrease of the droplet temperature and therefore to an increase of the surface tension. It was shown in [28] that the time of full evaporation of a droplet of the initial volume $7 \mu \mathrm{l}$ is about two times smaller under ambient air velocity of $1.2 \mathrm{~m} / \mathrm{s}$ than in quiescent air.

\subsection{Stages of evaporation}

Picknett and Bexon [10**] have been the first who pointed out two distinct modes or stages of evaporation - constant contact angle and constant contact line area. The comprehensive discussion on the subject is given in the recent review [13*], therefore in this subsection we will mainly discuss the works not included in [13*]. 
In the case when a liquid completely wets the solid support two stages of spreading/evaporation can be distinguished [29**]. During the first stage the droplet spreads over the substrate with a final contact angle close to zero. This process is usually very fast, therefore evaporation can be neglected and the droplet volume can be considered as a constant. During the second stage the spreading becomes negligible and the evaporation is the dominating process. Theoretical analysis performed in [29**] provides expressions for the kinetics of spreading/evaporation, i.e. dependency of the contact line radius and contact angle on time. The theoretical results obtained are in a good agreement with available experimental data.

In the case of partial wetting the spreading/evaporation of a liquid droplet placed on a solid support proceeds in general through four stages [30*] as shown in Fig. 3. First, the spreading stage occurs, during which the droplet attains the maximum value of the base diameter and the contact angle reaches the value of advancing contact angle. For simple liquids this stage is very fast and evaporation can be neglected during it, therefore in most studies it is out of consideration and only three consequent stages of evaporation are taken into account [31*-33*]. It is noteworthy that the mentioned stages of evaporation were observed on substrates of various wetting properties including superhydrophobic micropillar-patterned ones [34]. During the first stage of evaporation the drop base radius remains constant, whereas the contact angle decreases to the value of receding contact angle. Therefore, the first stage of evaporation is a manifestation of a contact angle hysteresis [35]. During the second stage the contact angle remains constant, but the drop base radius decreases. During the last, third, stage of evaporation both, the contact angle and the drop base radius decrease until the drop vanishes.

A detailed theoretical treatment of the kinetics of evaporating droplet during the first and second stages of evaporation was performed in [16**]. The derived analytical expressions for dependencies of droplet volume, base radius and contact angle on time demonstrated a good agreement with available experimental data on spreading of a droplet of water on various substrates as shown in Fig. 4. It is necessary to note, that by an appropriate choice of dimensionless time the time dependencies either of contact angle or contact line radius fall on the same master curve [16**]. The same theory governs the kinetics of organic liquids as well [4*]. During the both, first and second, stages of evaporation the value $V^{2 / 3}$ ( $V$ is the volume of the droplet) decreases linearly with time $\left[4^{\star}\right]$.

Whereas the behaviour of liquid during the first two stages of evaporation has got adequate theoretical description, the driving forces and kinetics of the third stage is still to be understood. Noteworthy that the contact angle during this stage decreases far below the value of the receding contact angle $\left[30^{\star}, 32^{\star}, 33^{\star}\right.$ and references therein]. It was assumed in [30*] that the third stage is determined by the surface forces acting in the vicinity of the three-phase contact line. The experimental study performed in [32*] evidenced that the drop of water deposited on polymeric substrate was surrounded by a thin precursor film, of a width about $10 \mu \mathrm{m}$. Therefore it was 
concluded in [32*] that this precursor film can be a clue to understanding the essential changes in the contact angle during the third stage of evaporation.

Film-type protrusions attached to an evaporating droplet of water have been observed also in [33*]. These attached films appeared at the second stage of evaporation, after the contact line started to recede. Their width was close to that described in [32*], about $10 \mu \mathrm{m}$, but the shape was more wavy. The film thickness of

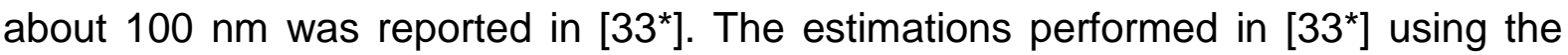
Cassie-Baxter model drive to the assumption that these attached films can be considered as one of the causes of a fast decrease of the contact angle during the third stage of evaporation. The rate of the contact angle decrease depended on the concentration of impurities in water.

\section{Effect of solid substrate}

It was already discussed above that the substrate thermo conductivity and thickness affect the surface temperature and in this way influence the evaporation kinetics. The substrate wetting properties are also of great importance. An increase in the roughness and/or chemical inhomogeneity of a substrate results in an increase of the contact angle hysteresis [35] having direct impact on the presence and duration of evaporation stages. By a high enough contact angle hysteresis the droplet evaporates mainly in the pinning mode, i.e. the stage with constant contact line radius prevails, whereas at a small contact angle hysteresis the contact line slips easily and the stage of constant contact angle is dominant.

For example, according to [36*] the evaporation of water droplets on metallic surfaces (steel, $\mathrm{Al}$ ) with a high contact angle hysteresis (up to 50 degrees) proceeds mainly in the regime with constant contact line radius (first stage of evaporation) which only in the very end is replaced by the regime with both, contact angle and contact line radius decreasing (third stage of evaporation in terms of Fig. 3). The metals used in [36*] were polished till a mean roughness of 4-8 $\mathrm{nm}$ and degreased, therefore the nature of a relatively high initial value of the contact angle (about 70 degree) and especially of a high contact angle hysteresis are to be explained. On polymeric surfaces with smaller contact angle hysteresis all three stages of evaporation were found, but the slip/stick motion of the contact line was observed during the second stage of evaporation [36*].

Dependence of the wetting behaviour on the substrate morphology has been shown also in [37] by studying the evaporation of droplets of water on four polymeric substrates of various hydrophobicity and morphology. By evaporation of aqueous droplets on micropillared superhydrophobic substrates the duration of the first stage of evaporation decreased and that of second stage increased by a decrease of solid fraction in the substrate area [34]. 
Comparison of the evaporation kinetics of ethanol droplets on three molecularly smooth substrates $\left(\mathrm{C}_{4} \mathrm{~F}_{8}\right.$, Cytop and PTFE) performed in [38*] has shown that the droplet life time depends essentially on hydrophobicity of the substrate. The three surfaces studied in [38*] were obtained by the same method - spin-coating on silicon wafers. The static contact angle of ethanol on $\mathrm{C}_{4} \mathrm{~F}_{8}$ was about 18 degrees and evaporation of ethanol droplet on this substrate proceeded in two stages - first with a constant contact line radius, but decreasing contact angle and second with a constant contact angle, but decreasing contact line radius. The static contact angle of ethanol on Cytop and Teflon was 45 and 57 degrees, respectively, and for both substrates there was no stage with decreasing contact angle, the contact angle remained equal to its static value whereas the contact line radius decreased all the time, i.e. no contact angle hysteresis was observed for ethanol on these substrates. The authors [38*] explain this difference in the behaviour of evaporating droplets by the fact that the "de-pinning" force is proportional to the $\sin \theta \cdot \delta \theta$. Therefore, at considerably smaller static contact angles much larger changes in the contact angle are necessary to de-pin the contact line. The similar behaviour was observed also for water droplets [39*].

According to [31*] the nature of the substrate can even affect the mechanism of dewetting. Studying evaporation of CTAB solutions on paraffin and Teflon surfaces by autoradiography method it was concluded in [31*] that depending on surfactant concentration and the substrate used dewetting occurs either by slipping or by "carpet rolling".

\section{Evaporation of complex liquids}

As equilibrium (advancing) contact angle depends on the liquid/air and solid/liquid interfacial tension, in the case of complex liquids the duration of the spreading stage can be determined by the adsorption kinetic of surfactants or nano-particles and therefore, it can be much longer than in the case of simple liquids. It was shown in [40] that for such surfactants as polyoxyethylene alkyl ethers and trisiloxanes at concentrations above 0.1 CAC (critical aggregation concentration, i.e. concentration above which self-assembled structures, such as micelles, vesicles etc. are spontaneously formed in solution) the spreading of small (several $\mu$ l) droplets is completed within about $100 \mathrm{~s}$. For this time evaporation is within $5 \%$ and therefore can be neglected. However, for example, for industrial surfactant Novec 4430 the equilibrium surface tension establishes very slowly even at concentrations near CAC (Fig. 5). That is why the spreading of solutions of this surfactant occurs also very slowly (Fig. 6). The results presented in Fig. 6 have been measured under $100 \%$ relative humidity, therefore evaporation can be neglected in this case. This example shows, that by studies on evaporation of complex liquids it should be taken into account that the stage of spreading can be considerably overlapped with the first stage of evaporation. 


\subsection{Aqueous surfactant solutions}

Surfactant can influence the evaporation kinetics of water in two different ways. First, the presence of surfactant slows down the evaporation because of i) decrease of molar fraction of water and ii) formation of adsorbed monolayer. The concentration of a surfactant in solutions is usually below $0.01 \mathrm{M}$, therefore such solutions can be considered as diluted and Raoult's law can be used to estimate the effect of surfactant on evaporation rate. Such estimation has been done in [30*] for SILWET L77 solution showing that for rather high concentration about $2 \mathrm{mM}$ (12.5 CAC) the change in the vapour pressure due to the presence of surfactant is less than $0.1 \%$. Therefore this effect can be neglected.

Some surfactant monolayers present on the interface can considerably suppress evaporation, but usually those are insoluble monolayers in condensed state [41]. The comprehensive study on adsorbed monolayers [42*] has shown that the only two from nine substances studied, namely sodium myristate and sodium palmitate have a distinct effect on water evaporation, whereas most of them, including SDS and CTAB do not influence it to a measurable degree. It is concluded in [42*] that evaporation can be hindered only by very densely packed adsorption layers.

The second effect is related to changes in the wetting properties due to surfactants. Addition of a surfactant to aqueous droplet facilitates evaporation of water, because it results in a decrease of the contact angle and therefore in an increase of the contact line radius $\left[30^{*}, 31^{*}, 43,44\right]$. The equilibrium contact angle decreases with the increase of surfactant concentration and levels off at a concentration called critical wetting concentration, CWC $\left[4^{*}, 43\right]$. For the surfactant solutions potentially capable to wet a substrate completely CWC is the concentration above which complete wetting occurs. CWC usually exceeds several times CAC despite the fact that the equilibrium surface tension reaches plateau at concentrations above CAC. The explanation of the difference between CWC and CAC still is to be found. It is also interesting that the value of CWC does not depend on the substrate used (i.e. it is the property of a surfactant). For surfactants facilitating complete wetting on moderately hydrophobic surfaces it occurs at the same concentration at which contact angle levels off on highly hydrophobic substrates [43].

If the initial surfactant concentration is lower than CWC then, during the evaporation process, due to decrease in the droplet volume and corresponding increase of the bulk concentration the equilibrium surface tension and the equilibrium contact angle will decrease until the bulk concentration reaches CWC what is illustrated by Fig. 7 [30*]. Transition from the first to the second stage of evaporation is seen clearly on curves 4 and 5 which correspond to concentrations equal and above CWC. At smaller concentrations one can see only a slight kink at the transition point, but the contact angle decreases further (curves 1-3 in Fig. 7). The curve 3, which 
corresponds to the concentration $\mathrm{CWC} / 2$, only in the very end of the time interval presented reaches the value of equilibrium contact angle corresponding to CWC and levels off. Noteworthy that the dependencies of contact line radius on time follow the pattern of Fig. 3 independently of concentration.

The theory describing kinetics of evaporation of pure liquids developed in [16*] was compared in [30*] with the experimental data for spreading/evaporation of droplet of SILWET L77 aqueous solution on Teflon as well as with the experimental data presented in [44] for spreading/evaporation of SDS solutions on Teflon. For both surfactants a very good agreement with the theoretical dependence of the contact angle on time has been obtained for the first stage of evaporation. The agreement between experimental data and the theoretical dependence of the contact line radius on time for the second stage of evaporation has been very good for concentrations above CWC. Although there was some deviation of experimental data from the theoretical curve for smaller concentrations, the agreement still was quite good even in this case. The dependence of the volume on time was the same for surfactant solutions as for pure liquids. Therefore, according to $\left[30^{\star}\right]$ the theory describing the first and second stages of evaporation of pure liquids is applicable for surfactant solutions as well despite the continuous decrease in the contact angle during the second stage of evaporation.

\subsection{Colloidal suspensions}

The presence of nano- or microparticles inside a liquid droplet can affect considerably the evaporation kinetics due to interaction of particles with the substrate and their adsorption on the liquid/air interface. Depending on particles and substrate properties, particles can accumulate at the liquid/air interface and/or liquid/substrate interface [45]. In particular, particles can deposit on the substrate near the threephase contact line (coffee ring effect [46*]) and keep the contact line pinned during the evaporation process increasing in this way the evaporation rate. An increase in nanoparticles concentration results in an increase of the pinning time and therefore in a decrease of the droplet lifetime $\left[39^{\star}, 47^{\star}\right]$. The pinning of the contact line by particles depends on hydrophobicity of the substrate. For highly hydrophobic substrates, where the droplets of pure liquids demonstrated the regime with constant contact angle and continuously decreasing contact line radius [38*, 39*], nanosuspensions demonstrated stick-slip behaviour with the duration of stick periods increasing with the increase in particles concentration [39*].

The stick-slip motion of the contact line is rather typical behaviour during the evaporation of a droplet of colloidal suspension. It is noteworthy, that both the pinned contact line and the "stick-slip" motion can be observed with the same suspension on the same substrate by only changing the environmental conditions, for example the pressure in the gas phase [48]. The single ring deposit with particulate coverage inside has been observed in the regime with pinned contact line, whereas the multiple concentric rings appeared after evaporation in the "stick-slip" regime. The 
very special case of "stick-slip" mode was observed in [49], where after the first and second stages of evaporation one more stage with pinned contact line was observed. In this case the deposit was formed mainly during this late "stick" stage.

Generally, the studies on evaporation of suspension droplets are in the most cases connected with the studies of deposits formed. Here we focus mostly on the evaporation kinetics, therefore the pattern formation is not discussed below. Detailed information on the last subject can be found in the very recent review [8*].

The rate of evaporation of a droplet of water decreases with the increase of the relative humidity of the ambient atmosphere. In the case of suspension this dependence can be modified if the contact angle and therefore the contact line radius of the suspension droplet depend on humidity. According to [50] this is the case for a whole blood droplet on a glass substrate. For the droplet of $14.2 \mu \mathrm{l}$, by an increase of the air relative humidity from 13.5 to $78 \%$ the initial contact angle decreased from 17 to 5 degrees, whereas the initial contact line diameter increased from 7.6 to $11.9 \mathrm{~mm}$. As a consequence the initial evaporation rate was independent of the relative humidity in this study [50]. The droplet was pinned through the whole evaporation process, therefore only the first stage of evaporation (Fig. 3) was observed.

The theory describing the evaporation kinetics of pure liquids [16**] was used in [51*] to analyse the evaporation kinetics of suspensions of $\mathrm{SiO}_{2}, \mathrm{TiO}_{2}$ and carbon nanoparticles on silicon wafers, Polyethylene and Teflon. Evaporation proceeded either in two stages or only in one stage (pinned mode), but in all cases a very good agreement with theory for pure liquids was found in [51*].

\section{Marangoni instability}

The heat transfer inside the evaporating droplet is of great importance because of its crucial impact on the evaporation kinetics. There is always Marangoni convection in evaporating droplet due to inhomogeneity of temperature which is taken into account in evaporation models $\left[16^{\star}, 52,53\right]$. Beside axisymmetric thermogravitational and thermocapillary convection, a more complicated convective motion in the form of cellular convection or longitudinal hydrothermal waves (HTWs) can develop inside the droplet as a result of Marangoni instability due to non-uniform temperature distribution, as was first demonstrated in [52*] and confirmed in the numerous consequent studies $\left[53,54^{\star}\right]$. The experimental study of these phenomena has been performed using infrared thermography. The dynamic regime in the droplet is timedependent. Three different regimes can be observed [54*]: i) warm up the droplet; ii) evaporation with acted convective instability; iii) evaporation without instability patterns. Instability type and characteristics depend essentially on the volatility of liquid used, which to a large extent governs the temperature gradients arising. For example, according to $[52,53]$ no instability has been detected by evaporation of 
water droplet; HTWs moving in azimuthal direction have been observed for more volatile methanol and ethanol; and convective cells emerging near the droplet apex and drifting to the edge developed when even more volatile Fluorinert FC-72 has been used. The number of waves in a more volatile liquid (methanol) has been larger in comparison to ethanol. It should be noted that according to [54*] the results obtained for water should be related only to a thin layer (about $0.2 \mathrm{~mm}$ ) below the water/air interface because of low transparency of water in infrared region.

The thermal conductivity and the temperature of substrate is also of importance for the temperature distribution over the droplet (especially near the TPL) and therefore for wave patterns observed. The number of waves observed increased with the increase of thermal conductivity $[52,53]$ and substrate temperature $\left[53,54^{\star}\right]$. The number of waves depends also on the droplet age, i.e. on its height. The smaller is the droplet height the smaller is the number of waves [52-54*].

For cellular convection (FC-72) the cells are larger near the droplet apex, where the temperature is lower. There is a region of small cells near the three-phase contact line. With time, as the droplet evaporate, the temperature gradient across the surface becomes smaller and the region occupied by small cells broadens $[52,53]$.

HTWs on the surface of evaporating ethanol droplet have been studied in [55] both under normal gravity and microgravity conditions. Waves move around the apex of a droplet and periodic changes of temperature are observed in azimuthal direction with a difference between the hot and cold points of about $1{ }^{\circ} \mathrm{C}$. Comparison of the results has shown that gravitational effects are insignificant for the hydrothermal waves development and the thermocapillary effect is responsible for the phenomenon.

The theoretical treatment of the convective instabilities accompanying the droplet evaporation is very complicated even in the frameworks of linear stability analysis because $\left[53,54^{\star}\right]$ : i) the problem is essentially 3-dimensional; ii) the profile of the droplet changes with time; iii) there is coupling between the heat transfer in three phases (solid substrate, liquid and vapour) and mass transfer in two fluid phases (liquid and vapour); and iv) evaporation rate depends on the solid/liquid contact angle. The first approach was proposed in [53] providing a detailed discussion on the problem statement, appropriate scaling, the dimensionless numbers involved and the choice of the perturbation functions enabling to follow the experimental trends.

\section{Conclusions}

Evaporation of liquid droplets is a quickly developing area nowadays, because it addresses both fundamental scientific problems and industrial interests. The recent studies have shown that evaporative cooling of droplet surface, properties of substrate and convection in the das phase can have considerable impact on the evaporation kinetics. The conditions have been outlined when and how such impact have to be taken into account. Essential progress has been reached in the 
understanding of evaporation of complex fluids, such as surfactant solutions and suspensions composed by micro- and nano-particles.

In our opinion evaporation of complex liquids is one of the priority areas for the nearest future, because it opens wide new possibilities for controlled formation of self-assembled structures of desired topology on micro- and nano-scale. The problems to be pointed out are as follows.

1. It was shown recently that theory describing kinetics of the evaporation of pure liquids in the case of partial wetting can be successfully applied to surfactant solutions and suspensions as well. There is a theory developed for the simultaneous spreading and evaporation of pure liquids in the case of complete wetting, but no experimental data related to the case for the complex liquids. Obviously this gap should be filled.

2. There are practically no results on spreading/evaporation of non-Newtonian liquids. Therefore efforts are expected to be undertaken in this area both in theory and in experimental studies.

3. There is a lot of results published on the pattern formation during evaporation of the complex liquids, but there is no predictive theory yet, enabling the full control over this process. The further quick development in this area is expected.

4. The fast development can be predicted on the research of evaporation of biological liquids in particular to develop diagnostic tools.

\section{References}

* of special interest

** of outstanding interest

1. Semenov, S., Starov, V.M., Velarde, M.G., Rubio, R.G. Droplets evaporation: problems and solutions. Eur. Phys. J. Special Topics 2011;197:265-278.

2. Holyst, R., Litnievski, M., Jakubczyk, D., Kolwas, K., Kolwas, M., Kowalski, K., Migacz, S., Palesa, S., Zientara, M. Evaporation of freely suspended single droplets: experimental, theoretical and computational simulations. Rep. Prog. Phys. 2013;76:034601.

3. Sazhin, S.S. Advanced models of fuel droplet heating and evaporation. Progress in Energy and Combustion science 2006;32:162-214.

4*. Semenov, S., Trybala, A., Rubio, R.G., Kovalchuk, N., Starov, V., Velarde, M.G. Simultaneous spreading and evaporation: Recent developments. Adv. Colloid Interface Sci. 2014;206:382-398. 
5*. Erbil, H.Y. Evaporation of pure liquid sessile and spherical suspended drops: A review. Adv. Coll. Int. Sci. 2012:170;67-86.

6*. Sefiane, K., Skilling, J., MacGillivray, J. Contact line motion and dynamic wetting of nanofluid solutions. Adv. Coll. Int. Sci. 2008;138:101-120.

7. Stannard, A. Dewetting-mediated pattern formation in nano-particle assemblies. J. Phys.: Condens. Matter 2011;23:083001.

8*. Thiele, U. Patterned deposition at moving contact lines, Adv. Coll. Int. Sci., 2014;206:399-413.

9. Mijatovic, D., Eijkel, J. C. T., van den Berg, A. Technologies for nanofluidic systems: top-down vs. bottom-up - a review. Lab Chip, 2005;5:492-500.

10**.Picknett, R.G., Bexon, R. The evaporation of sessile or pendant drops in still air. J. Coll. Int. Sci. 1977, 61, 336-350.

$11^{\star \star}$. Hu, H., Larson, R.G. Evaporation of a sessile droplet on a substrate. J. Phys. Chem. B 2002; 106: 1334-1344.

$12^{\star \star}$. Sefiane, K., Bennacer, R. An expression for droplet evaporation incorporating thermal effects. J. Fluid. Mech. 2011; 667: 260-271.

13*. Cazabat, A.-M., Guena, G. Evaporation of macroscopic sessile droplets. Soft Matter 2010; 6: 2591-2612.

14. Semenov, S., Starov, V.M., Rubio, R.G., Velarde, M.G. Computer simulations of evaporation of pinned sessile droplets: Influence of kinetic effects. Langmuir 2012; 28: 15203-15211.

15*. Saada, M.A., Chikh, S., Tadrist, L. Evaporation of a sessile drop with pinned or receding contact line on a substrate with different thermophysical properties. Int. J. Heat Mass Transfer 2013; 58: 197-208.

16**. Semenov, S., Starov, V.M., Rubio, R.G., Agogo, H., Velarde, M.G. Evaporation of sessile water droplets: Universal behaviour in presence of contact angle hysteresis. Coll. Surf. A 2011; 391:135-144.

17*. Lopes, M.C., Bonaccurso, E., Gambaryan-Roisman, T. Stephan, P. Influence of substrate thermal properties on sessile droplet evaporation: Effect of transient heat transport. Coll. Surf. A 2013;432:64-70.

18. David, S., Sefiane, K., Tadrist, L. Experimental investigation of the effect of thermal properties of the substrate in the vetting and evaporation of sessile drops. Coll. Surf. A 2007; 298: 108-114.

19. Starov, V., Sefiane, K. On evaporation rate and interfacial temperature of volatile sessile drops. Coll. Surf. A 2009; 333: 170-174. 
20*. Semenov, S., Starov, V.M., Rubio, R.G., Velarde, M.G. Instantaneous distribution of fluxes in the course of evaporation of sessile liquid droplets: computer simulations. Coll. Surf. A 2010; 372: 127-134.

21. Gelderblom, H., Martin, A.G., Nair, H., van Houselt, A., Lefferts, L., Snoeijer, J.H., Lohse, D. How water droplets evaporate on a superhydrophobic substrate. Phys. Rev. E 2011; 83: 026306.

22*. Sobac, B., Brutin, D. Triple line behaviour and wettability controlled by nanocoated substrates: Influence on sessile drop evaporation. Langmuir 2011;27:14999-15007.

23. Sobac, B., Brutin, D. Thermal effects of the substrate on water droplet evaporation. Phys. Rev. E 2012;86:021602

24*. Saada, M.A., Chikh, S., Tadrist, L. Numerical investigation of heat and mass transfer of an evaporating sessile drop on a horizontal surface. Phys. Fluids 2010;22:112115.

$25^{\star \star}$. Carle, F., Sobac, B., Brutin, D. Experimental evidence of the atmospheric convective transport contribution to sessile droplet evaporation. Appl. Phys. Lett. 2013;102:061603

26. Kelly-Zion, P.L., Pursell, C.J., Vaidya, S., Batra, J. Evaporation of sessile drops under combined diffusion and natural convection. Coll. Surf. A 2011; 281: 31-36.

27. Kelly-Zion, P.L., Pursell, C.J., Hasbamrer, N., Cardozo, B., Gaughan, K., Nickels, K. Vapour distribution above an evaporating sessile drop. Int. J. Heat Mass Transfer 2013;65:165-172.

28. Bin, L., Bennacer, R., Bouvet, A. Evaporation of methanol droplet on the Teflon surface under different air velocities. Appl. Thermal Eng. 2011; 2011:37923798.

29**. Lee, K.S., Cheah, C.Y., Copleston, R.J., Starov, V.M., Sefiane, K. Spreading and evaporation of sessile droplets: Universal behavior in the case of complete wetting. Coll. Surf. A 2008; 323: 63-72.

30*. Semenov, S., Trybala, A., Agogo, H., Kovalchuk, N., Ortega, F., Rubio, R.G., Starov, V.M., Velarde, M.G. Evaporation of droplets of surfactant solutions. Langmuir 2013; 29:10028-10036.

31*. Soboleva, O.A., Summ, B.D. The kinetics of dewetting of hydrophobic surfaces during the evaporation of surfactant solution drops. Coll. J. 2003; 65: 8993. 
32*. Bormashenko, E., Bormashenko, E., Whyman, G., Pogreb, R., Musin, A., Jager, R., Barkay, Z. Contact angle hysteresis on polymer substrates established with various experimental techniques, its interpretation, and quantitative characterisation. Langmuir 20088; 24: 4020-4025.

33*. Park, J.K., Ryu, J., Koo, B.C., Lee, S., Kang, K.H. How the change of contact angle occurs for an evaporating droplet: effect of impurity and attached water films. Soft Matter 2012; 8: 11889-11896.

34. Xu, W., Leeladhar, R., Kang, Y.T., Choi, C.-H. Evaporation kinetics of sessile water droplets on micropillared superhydrophobic surfaces. Langmuir 2013; 29: 6032-6041.

35. Eral, H.B., 't Mannetje, D.J.C.M., Oh, J.M. Contact angle hysteresis: a review of fundamentals and applications. Colloid Polym. Sci. 2013; 291: 247-260.

36*. Bormashenko, E., Musin, A., Zinigrad, M. Evaporation of droplets on strongly and weakly pinning surfaces and dynamic of triple line. Coll. Surf. A 2011; 385: 235-240.

37. Pittoni, P.G., Chang, C.-C., Yu, T.-S., Lin, S.-Y. Evaporation of water drops on polymer surfaces: Pinning, depinning and dynamics of the triple line. Coll. Surf. A 2013; 432: 89-98.

38*. Shanahan, M.E.R., Sefiane, K, Moffat, J.R. Dependence of volatile droplet lifetime on the hydrophpbicity of the substrate. Langmuir 2011; 27: 4572-4577.

39*. Orejon, D., Sefiane, K., Shanahan, M.E.R. Stick-slip of evaporating droplets: substrate hydrophobicity and nanoparticle concentration. Langmuir 2011; 27 : 12834-12843.

40. Ivanova, N.A., Zhantenova, Zh.B., Starov, V.M. Wetting dynamics of polyoxyethylene alkyl ethers and trisiloxanes in respect of polyoxiethylene chains and properties of substrates. Col. Surf. A 2012; 413: 307-313.

41. Barnes, G.T. The potential for monolayers to reduce the evaporation of water from large water storages. Agricultural Water Management 2008; 95: 339-353.

42*. Lunkenheimer, K., Zembala, M. Attempts to study a water evaporation retardation by soluble surfactants. J. Coll. Int. Sci. 1997; 188: 363-271.

43. Ivanova, N., Starov, V., Rubio, R., Ritacco, H., Nilal, N., Johnson, D. Critical wetting concentrations of trisiloxane surfactants. Coll. Surf. A 2010; 354: 143-148.

44. Doganci, M.D., Sesli, B.U., Erbil, H.Y. Diffusion controlled evaporation of sodium dodecyl sulphate solution drops placed on a hydrophobic substrate. J. Coll. Int. Sci. 2011; 362: 524-531. 
45. Trantum, J.R., Eagleton, Z.E., Patil, C.A., Tucker-Schwartz, J.M., Baglia, M.L., Skala, M.C., Haselton, F.R. Cross-sectional tracking of particle motion in evaporating drops:flow fields and interfacial accumulation. Langmuir 2013; 29: 6221-6231.

46*. Deegan, R.D., Bakajin, O., Dupont, T.F., Huber, G., Nagel, S.R., Witten, T.A. Capillay flow as the cause of ring stains from dried liquid drops. Nature 1997; 389: 827-829.

47*. Nguyen, T.A.H., Nguyen, A.V. Increased evaporation kinetics of sessile droplets by using nanoparticles. Langmuir 2012; 28: 16725-16728.

48. Ascounis, A., Sefiane, K., Koutsos, V., Shanahan, M.E.R. The effect of evaporation kinetics on nanoparticle structuring within contact line deposits of volatile drops. Coll. Surf. A 2014; 441: 855-866.

49. Nguyen, T.A.H., Hampton, M.A., Nguyen, A.V. Evaporation of nanoparticle droplets on smooth hydrophobic surfaces: the inner coffee ring deposits. J. Phys. Chem. C 2013; 117: 4707-4716.

50. Bou Zeid, W., Brutin, D. Influence of relative humidity on spreading, pattern formation and adhesion of a drying drop of whole blood. Coll. Surf. A 2013; 430: 1-7.

51*. Trybala, A., Okoye, A., Semenov, S., Agogo, H., Rubio, R.G., Ortega, F., Starov, V.M. Evaporation kinetics of sessile droplets of aqueous suspensions of inorganic nanoparticles. J. Coll. Int. Sci. 2013; 403: 49-57.

52*. Girard F., Antoni M., Faure S., Steinchen A. Evaporation and Marangoni driven convection in small heated water droplets. Langmuir 2006; 22: 1108511091.

53. Girard F., Antoni M., Sefiane K. On the effect of Marangoni flow on evaporation rates of heated water dropls. Langmuir 2008; 24: 9207-9210.

54. Sefiane, K., Moffat, J.R., Matar, O.K., Craster, R.V. Self-excited hydrothermal waves in evaporating sessile drops. Applied Phys. Let. 2008; 93: 074103.

55. Sefiane, K., Steinchen, A., Moffat, R. On hydrothermal waves observed during evaporation of sessile droplets. Coll. Surf. A 2010; 365: 95-98.

56*. Brutin, D., Sobac, B., Rigollet, F., Le Niliot, C. Infrared visualization of thermal motion inside a sessile drop deposited onto a heated surface. Exp. Thermal and Fluid Sci. 2011; 35: 521-530.

57. Carle, F., Sobac, B., Brutin, D. Hydrothermal waves on ethanol droplets evaporating under terrestrial and reduced gravity levels. J. Fluid. Mech. 2012; 712: 614-623. 


\section{Figure captions}

Fig. 1. Dependence of the evaporation rate of sessile droplet normalised by the evaporation rate in isothermal conditions, $\boldsymbol{M}$, on Sefiane-Bennacer number: $a_{1}=\frac{\left(\partial c_{s} / \partial T\right)_{T \infty}}{\left(c_{s}\right)_{T \infty}}, \dot{m}_{d} / \pi r_{d}=D c_{s}\left(T_{\infty}\right), \mathcal{L}$ is the latent heat of evaporation, $k_{L}$ and $k_{S}$, are thermo conductivities of liquid and solid respectively, $r_{e d g} \theta$ and $I_{S}$ are the effective thicknesses of liquid and solid respectively. Adopted from [12**].

Fig. 2. Evaporation rate of an ethanol sessile droplet as a function of the temperature difference between the substrate and the ambient air for terrestrial and microgravity conditions $\left[25^{\star \star}\right]$.

Fig. 3. Spreading stage and the three stages of evaporation in the case of partial wetting [30*]. Lad is the maximum radius of the contact line, $\theta_{\text {ad }}$ is the contact angle corresponding to the time when Lad is reached (beginning of the Stage 1), $\theta_{r}$ is the constant value of the contact angle during the Stage II.

Fig. 4. Kinetics of spreading of water drop on: 1 - polished Epoxy, 2 - Corning glass 7740, 3 - PMMA, 4 - PET; a - first stage of evaporation (constant drop base radius), $\mathrm{b}$ - second stage of evaporation (constant contact angle); $\ell$ is the dimensionless contact line radius normalised by the maximum contact line radius, $\tau$ is the dimensionless time, $\tau_{r}$ is the dimensionless time corresponding to the beginning of the second stage (see $\left[16^{\star \star}\right]$ for details). Adopted from [16**].

Fig. 5. Dynamic surface tension of Novec 4430 solution: $1-0.015 \mathrm{~g} / \mathrm{l}, 2-0.25 \mathrm{~g} / \mathrm{l}$. $\mathrm{CAC} \sim 0.2 \mathrm{~g} / \mathrm{l}$.

Fig. 6. Kinetics of spreading of Novec 4430 solution, concentration $0.5 \mathrm{~g} / \mathrm{l}$.

Fig. 7. Time dependence of the contact angle on Teflon for aqueous solution of SILWET L77 surfactant at concentrations: $1-\mathrm{c}=0.07 \mathrm{~g} / \mathrm{l}, 2-\mathrm{c}=0.09 \mathrm{~g} / \mathrm{l}, 3-\mathrm{c}=0.125$ $\mathrm{g} / \mathrm{l}, 4-\mathrm{c}=0.25 \mathrm{~g} / \mathrm{l}(\mathrm{CWC}), 5-\mathrm{c}=0.8 \mathrm{~g} / \mathrm{l}$. Measurement is performed at $24^{\circ} \mathrm{C}$ and $50 \%$ relative humidity, the third stage of evaporation is not shown [30*]. 
Figures

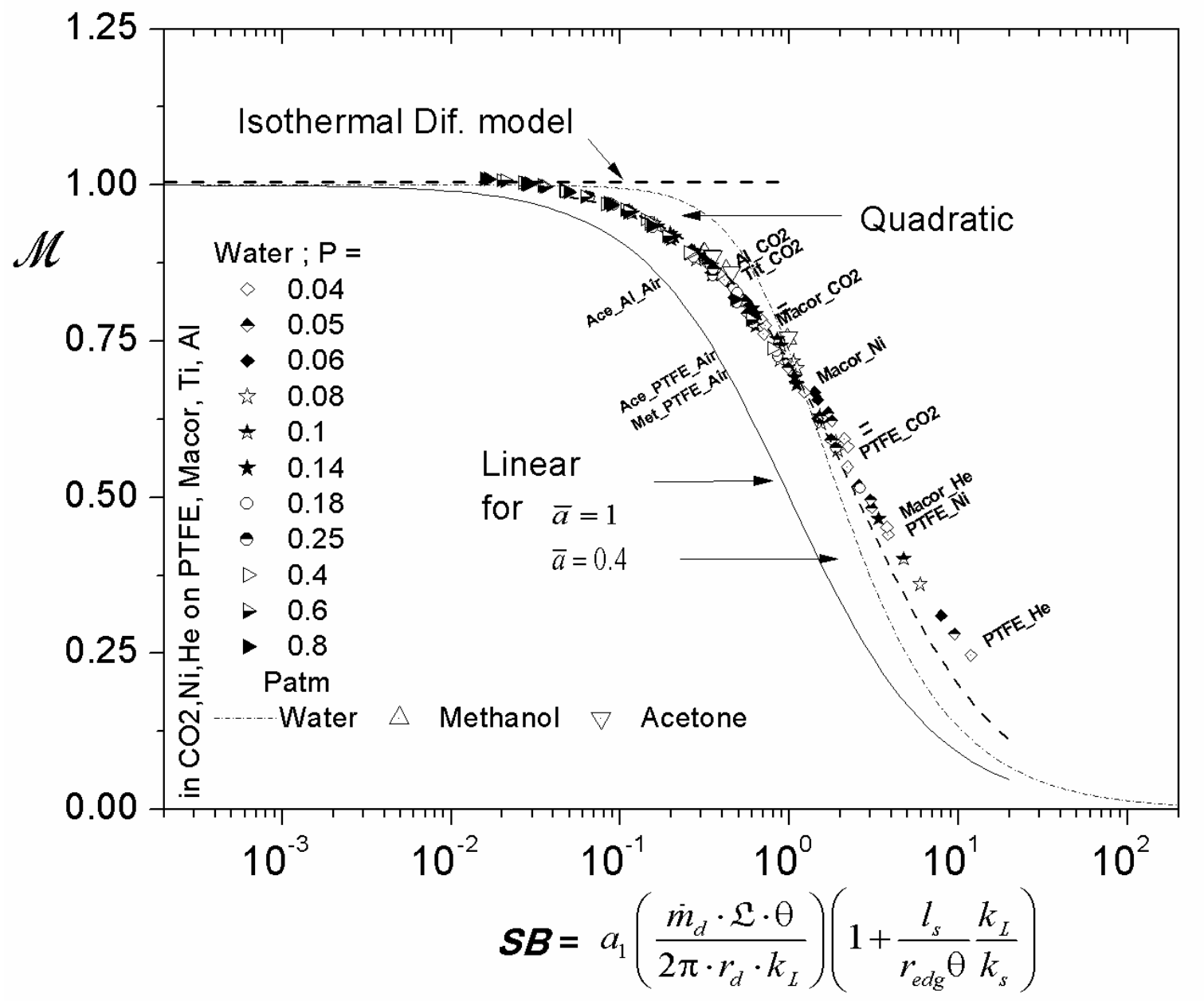

Fig. 1. Dependence of the evaporation rate of sessile droplet normalised by the evaporation rate in isothermal conditions, $\boldsymbol{M}$, on Sefiane-Bennacer number: $a_{1}=\frac{\left(\partial c_{s} / \partial T\right)_{T \infty}}{\left(c_{s}\right)_{T \infty}}, \dot{m}_{d} / \pi r_{d}=D c_{s}\left(T_{\infty}\right), \mathcal{L}$ is the latent heat of evaporation, $k_{L}$ and $k_{s}$, are thermo conductivities of liquid and solid respectively, $r_{e d g} \theta$ and $I_{s}$ are the effective thicknesses of liquid and solid respectively. Adopted from [12**]. 

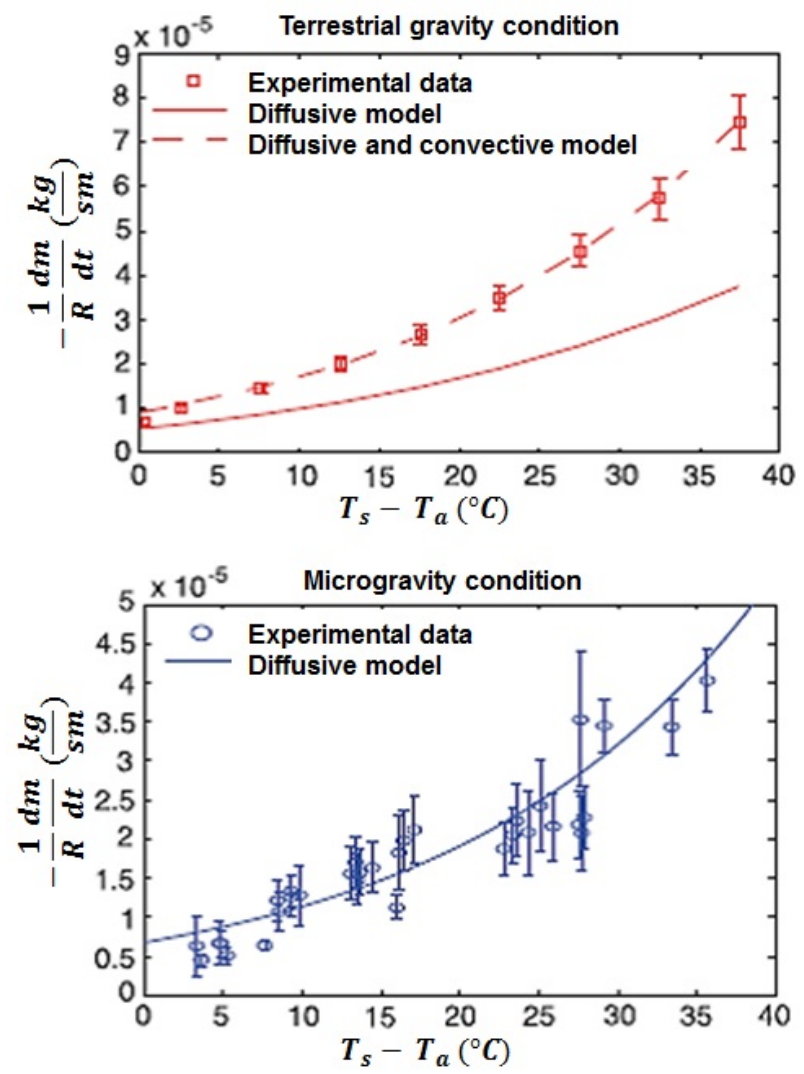

Fig. 2. Evaporation rate of an ethanol sessile droplet as a function of the temperature difference between the substrate and the ambient air for terrestrial and microgravity conditions $\left[25^{\star \star}\right]$. 


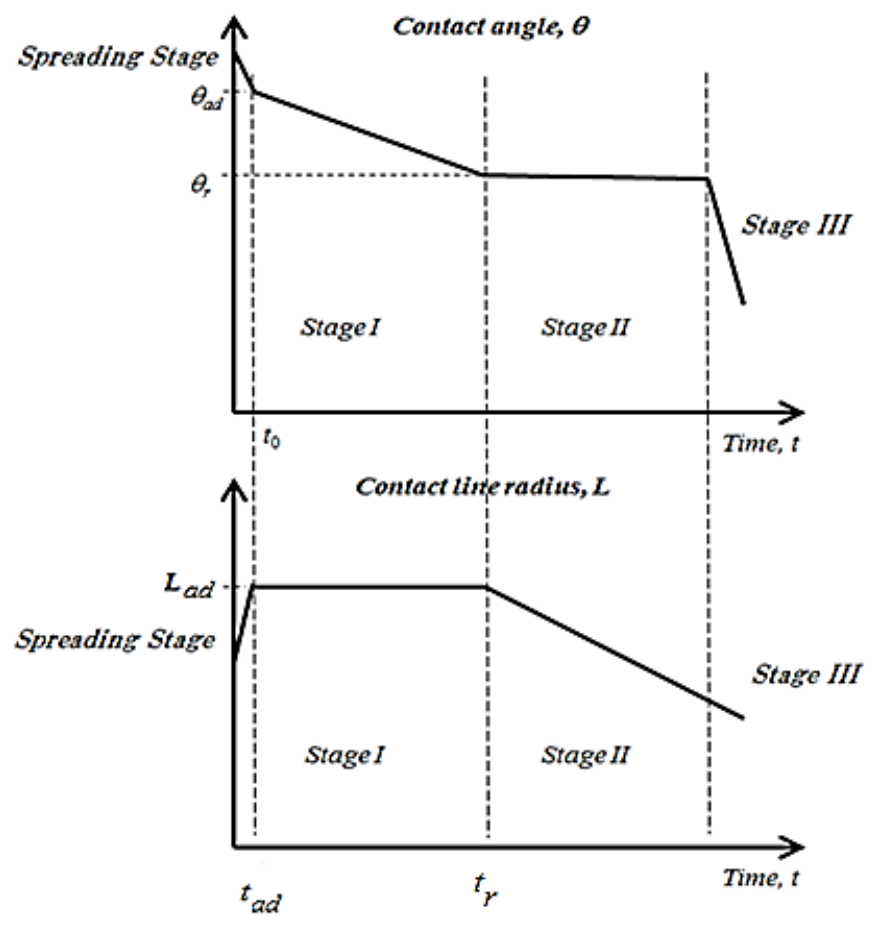

Fig. 3. Spreading stage and the three stages of evaporation in the case of partial wetting [30*]. Lad is the maximum radius of the contact line, $\theta$ ad is the contact angle corresponding to the time when $L_{a d}$ is reached (beginning of the Stage 1 ), $\theta_{r}$ is the constant value of the contact angle during the Stage II.

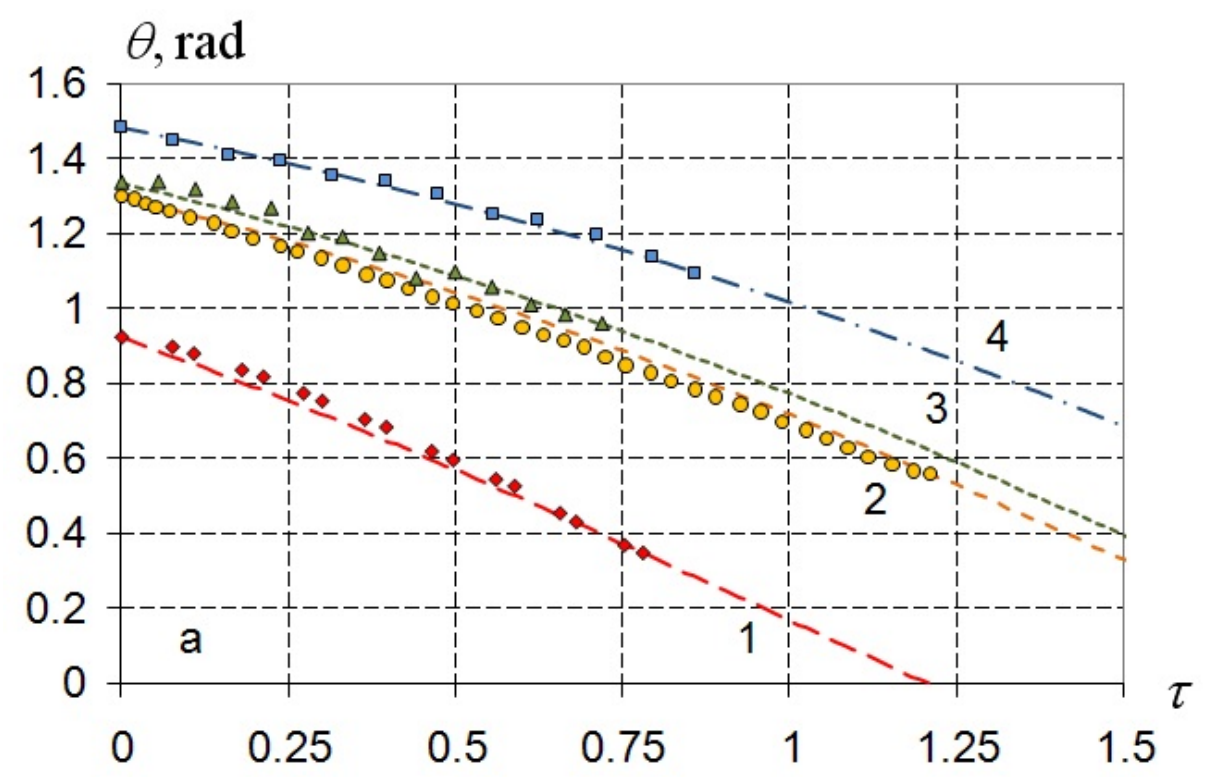




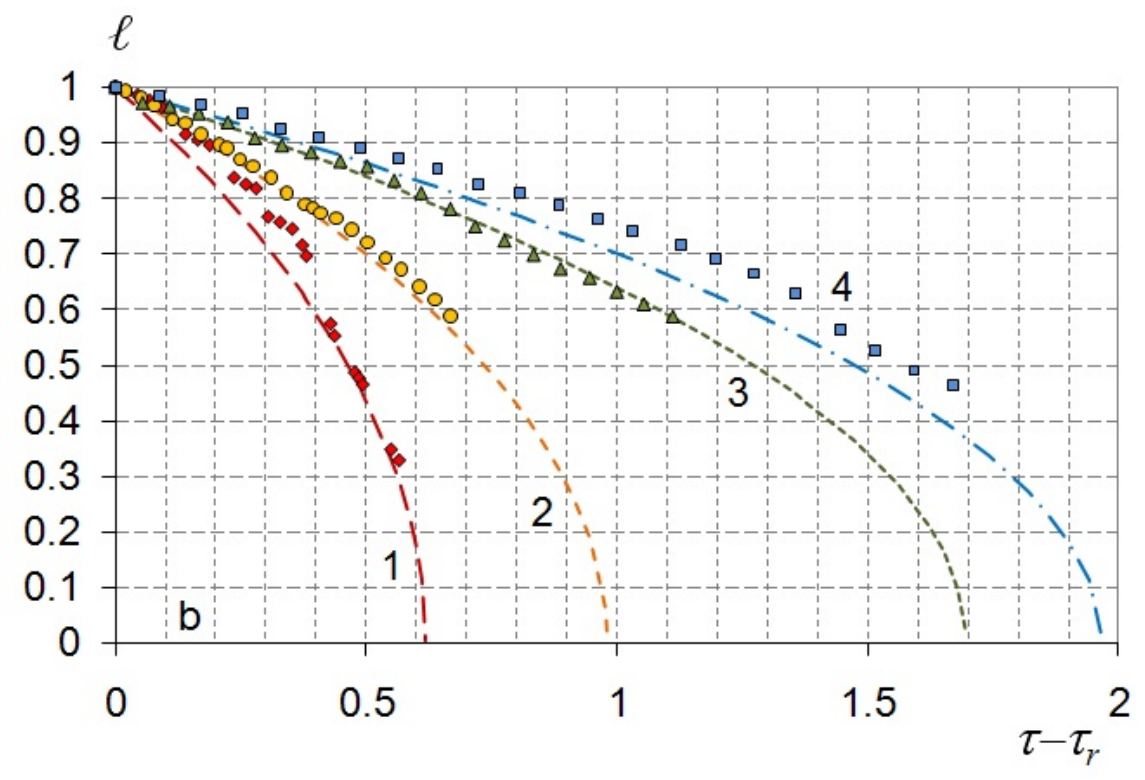

Fig. 4. Kinetics of spreading of water drop on: 1 - polished Epoxy, 2 - Corning glass 7740, 3 - PMMA, 4 - PET; a - first stage of evaporation (constant drop base radius), $\mathrm{b}$ - second stage of evaporation (constant contact angle); $\boldsymbol{l}$ is the dimensionless contact line radius normalised by the maximum contact line radius, $\tau$ is the dimensionless time, $\tau_{r}$ is the dimensionless time corresponding to the beginning of the second stage (see $\left[16^{\star \star}\right]$ for details). Adopted from $\left[16^{\star \star}\right]$.

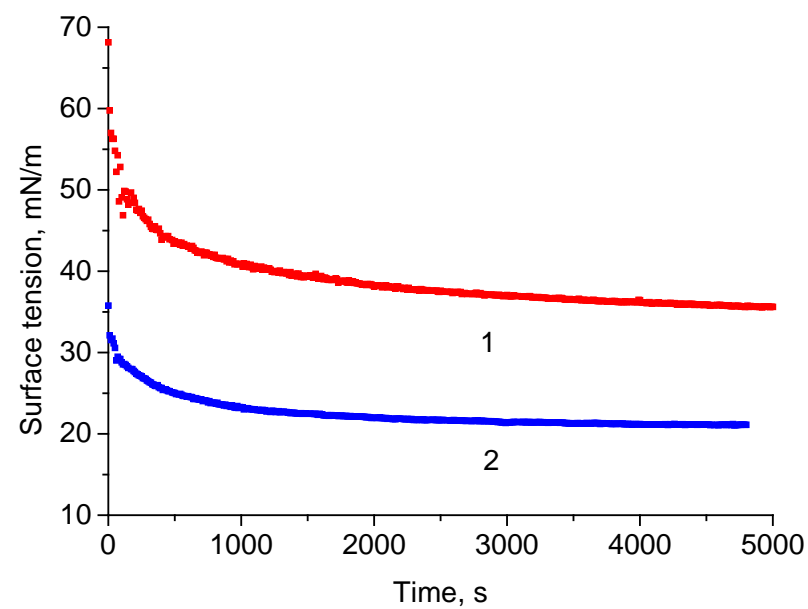

Fig. 5. Dynamic surface tension of Novec 4430 solution: $1-0.015 \mathrm{~g} / \mathrm{l}, 2-0.25 \mathrm{~g} / \mathrm{l}$. $\mathrm{CAC} \sim 0.2 \mathrm{~g} / \mathrm{l}$. 


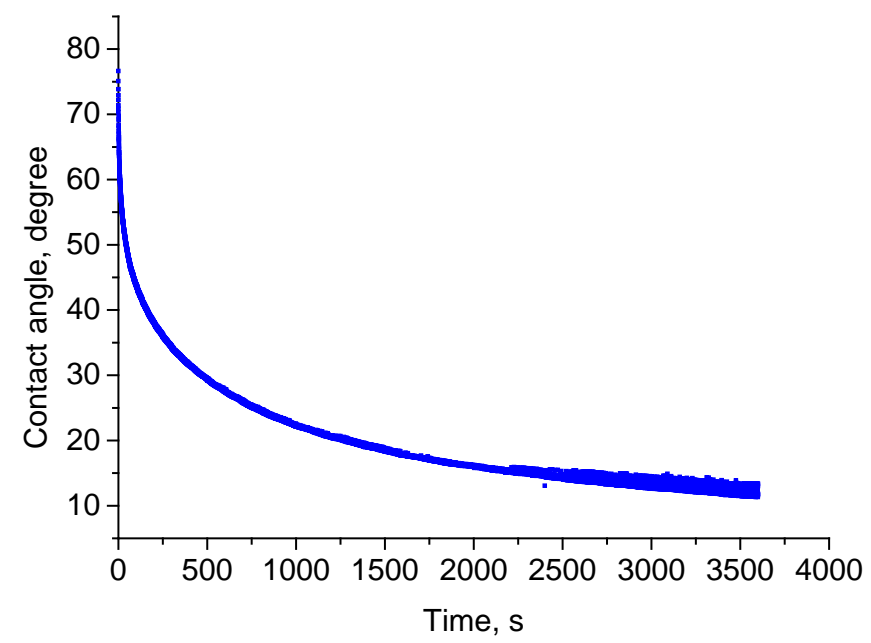

Fig. 6. Kinetics of spreading of Novec 4430 solution, concentration $0.5 \mathrm{~g} / \mathrm{l}$.

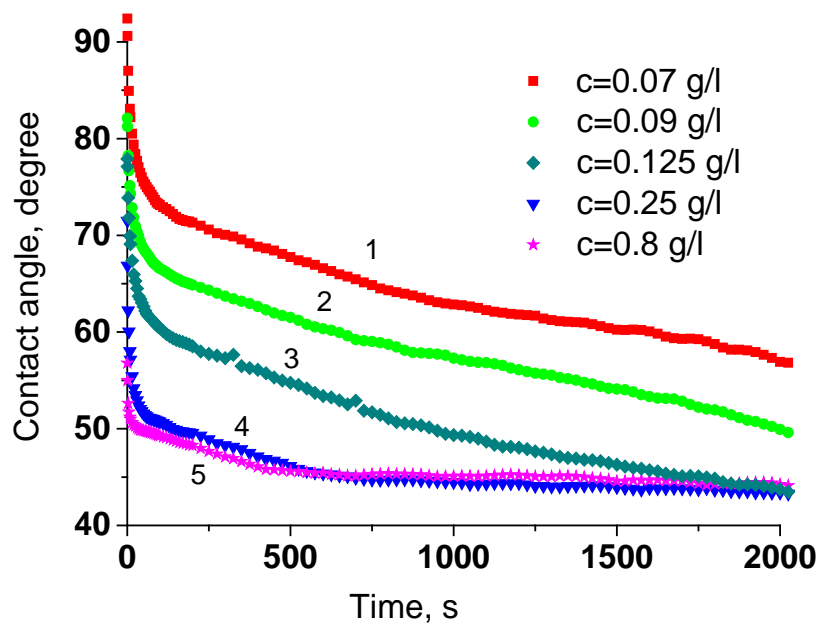

Fig. 7. Time dependence of the contact angle on Teflon for aqueous solution of SILWET L77 surfactant at concentrations: $1-\mathrm{c}=0.07 \mathrm{~g} / \mathrm{l}, 2-\mathrm{c}=0.09 \mathrm{~g} / \mathrm{l}, 3-\mathrm{c}=0.125$ $\mathrm{g} / \mathrm{l}, 4-\mathrm{C}=0.25 \mathrm{~g} / \mathrm{l}(\mathrm{CWC}), 5-\mathrm{C}=0.8 \mathrm{~g} / \mathrm{l}$. Measurement is performed at $24^{\circ} \mathrm{C}$ and $50 \%$ relative humidity, the third stage of evaporation is not shown [30*]. 